\title{
Implementation Of Ontology Matching Using Protégé
}

$\begin{array}{ccccc}\text { Jayanth Kumar } & \text { Sujitha Reddy } & \text { Priya.M } & \text { Raju.J } & \text { Vanmathi.C } \\ \text { SITE } & \text { SITE } & \text { SITE } & \text { SITE } & \text { SITE } \\ \text { VIT University } & \text { VIT University } & \text { VIT University } & \text { VIT University } & \text { VIT University } \\ \text { Vellore, India } & \text { Vellore, India } & \text { Vellore, India } & \text { Vellore, India } & \text { Vellore, India }\end{array}$

Abstract: Ontologies play an important role for interoperability between organizations and for semantic web because they aim at capturing domain knowledge. Different solutions exist to identify the common concepts and involve different sources using ontology. Matching is a key operation in semantic web since it takes inputs as ontologies; each consists of a set of discrete entities like tables, xml elements, classes, properties. In this paper, we developed two anthologies by which we matched those ontologies to get the similar classes, objects, instances by using Protégé. Protégé is a tool used for comparing ontologies, merging ontologies, data translation, query processing.

Keywords: Ontology,Ontology Matching,Merging Ontologies,Domain Knowledge

\section{INTRODUCTION}

An ontology is a broader knowledge model with a reasoning mechanism that facilitates knowledge on the semantic web [6]. Semantic web represents web content in a form that is easily machine process- able. It uses intelligent techniques to take advantage of these representations. Semantic web knowledge will be organized in conceptual spaces according to meaning. Semantic web technologies are Ontologies, Explicit Metadata, Logic and Inference and Agents [6]. The term ontology originates from a philosophy which means nature of existence

T.R Gruber's definition was redefined by R.Studer as "Ontology is an explicit and formal specification of a conceptualization" [3].

The knowledge representation languages are used to create sets of terms and axioms about the meanings of the terms as well as to specify classes, properties and relationships between classes and objects in a domain. Reasoning engines like Fact++, Hermi 1.2.8, DIG and Pellet 1.2.8 are used to infer the meanings and relationships between the classes, subclasses, and superclasses It will build a taxonomic structure [4] [5] and checks the model consistency.

Ontology for the semantic web is typically constructed in notational languages like RDF, RDFS and OWL.

RDF is Resource Description Framework and it is used for describing resources on the web. It is designed to understand and read by computers. This is written in XML and it is a part of $\mathrm{W} 3 \mathrm{C}$ semantic web activity. RDF identifies things using web identifiers and describes the resources with properties, property values.

RDFS is a Resource Description Framework Schema. It does not provide actual application specific classes and properties. Classes in RDFS are like classes in object oriented programming, so it is easy for resources to define instances of classes, and subclasses of classes.

OWL is a web ontology language. It is built on top of RDF and is used for processing information on the web. It has been designed and interpreted by computers and not for people. Owl and RDF are one on the same thing but OWL is stronger language with the more interpretability than RDF. It comes with a larger vocabulary and a stronger syntax than RDF.

OWL has three types of languages

- $\quad$ OWL LITE

- OWL DL

- OWL FULL

Many applications such as data integration, ecommerce and semantic web services, which are exploiting internet content represented in a form that is easily processed by machine and enriched with metadata. Ontologies play an important role for interoperability between organizations and for semantic web because they aim at capturing domain knowledge in a generic way and provide a proper understanding of the domain [7] [8].

For developing the ontologies we need to go through these notational languages.

With the developed ontologies we can go with different types of operations like ontology matching, ontology merging, query processing and many more.

An ontology matching is like developing ontology and compares those two by which we will get similar classes, instances and properties of a class.

Ontology is considered here as taxonomy of concepts and the problems of matching are reduced to "for each concept node in one taxonomy, find the most similar node in the other taxonomy". Our concern discussed in this paper is to make corrections to eliminate the matching concepts.

The mapping is the most important step in Ontology alignment and it is described informally as where for each entity in one ontology we try to find a same entity in the second anthology, with the same meaning. The result of the mapping may be as simple as one-one correspondences between some concepts and they can be declarative concepts.

After some years people found tools for developing ontologies without using the notational If they are trying to develop ontologies automatically the code will be generated by using these tools, so need to go with the notational languages nowadays. 
There are several tools which help in developing ontologies: [1] [2]

$\begin{array}{ll}- & \text { Protégé } \\ \text { - } & \text { Swoop } \\ \text { - } & \text { Top Braid composer } \\ \text { - } & \text { Oiled } \\ \text { - } & \text { OnebODE } \\ \text { - } & \text { Intolingua } \\ \text { - } & \text { Onto track } \\ - & \text { IHMC } \\ - & \text { Cmap Ontology editor }\end{array}$

- $\quad$ By using these tools we can define new concepts, relations and instances. These tools may contain additional features such as graphical browsing, search and constraint checking capabilities

\section{RELATED WORK}

a. Hacene Belhadefa [2011], detected and repaired the list of homologs concepts; which is based on the bidirectional comparison (Checking) between the two matched ontologies to filter this list of concepts. The measures used are classical just to show the reliability and reliability of their method, but they generalized it for supporting other advanced measures. Here an ontology is considered here as a taxonomy of concepts and the problem of matching is reduced to "for each concept node in one taxonomy, find the most similar node in the other taxonomy". Their main concern is to discuss in their paper is the reliability of the results and how to make corrections to eliminate the matching concepts when the degree is low [1].

b. Haridimos Kondylakis [et. al] presented a mapping language for information integration under a common knowledge representation model (LAV approach). They proposed a specific mapping annotation format that is capable to capture all those cases. They assume that the level of detail of this format is sufficient to produce complete mediation of data transformation algorithms without further input from the domain experts. They noted that their mappings will be used like in a Local as View mediation process and the mapping format should give enough specifications to an IT-expert to start building an integration algorithm without any help from the domain expert. Their plans for future work include the task of verification of their mapping mechanism [7]

c. M. Rahamatullah Khondoker,[et.al] Finded an appropriate tool to develop ontology is the first step towards ontology development in a lot of ontology development tools are available in the market, however, some are free and some are commercial. Ontology development is a complex and largely domain-oriented process that can be benefited from tool support. Ontology development tools are compared based on certain features such as modelling features/limitations, base language, web support and use, import/export format, graph view, consistency checks, multi-user support, merging, lexical support, and information extraction. Compares development tools based on userexperience is a scarce attention from the research community

\section{ONTOLOGY MATCHING}

Ontology alignment or ontology matching is the process of determining correspondences between concepts [3] [4]. A set of correspondences is also called as alignment. Ontology matching is the best solution for the heterogeneity problem because it finds correspondences between semantically related entities of the ontologies. These correspondences can be used for various tasks like ontology merging, query answering, and data translation and for navigation on the semantic web.The matching ontologies enable the knowledge and data expressed in the matched ontologies to interoperate.The alignment of two ontologies, Ontology1 and Ontology2 amounts to determining the correspondence between the various ontological entities by category. All methods of alignment determine the correspondences between ontological entities using measures of similarity. The measures of similarity or dissimilarity allow evaluating the similarity or the distance between two elements or (individuals).Subsequent Pages

\section{IMPLEMENTATION DETAILS}

Normally all the ontologies developed by using notational languages like XML, RDF, , OWL. By using these languages it is hard to implement or develop ontologies. To avoid them, tools are developed and one of them is Protégé which is good to develop ontologies as well as for matching ontologies to get the similar correspondences in a result. [5]

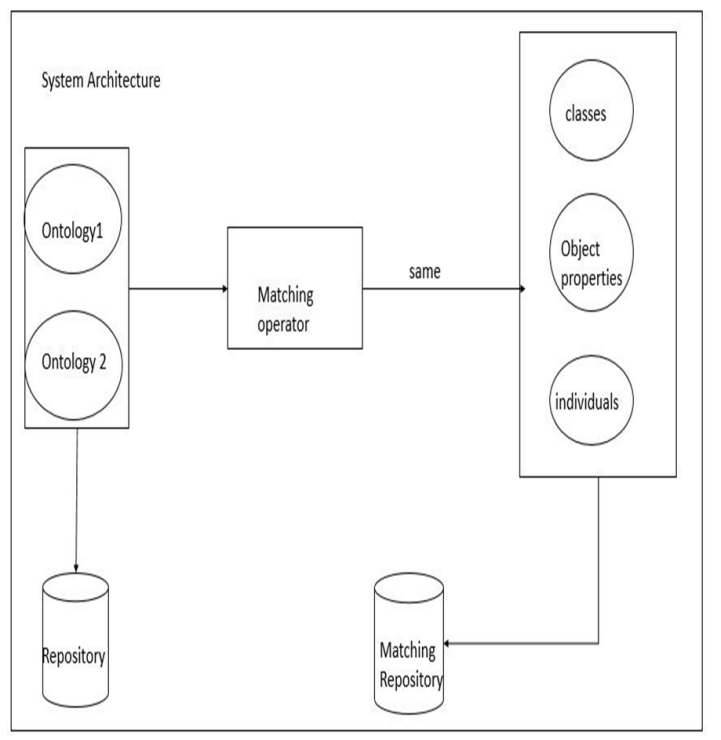

Fig 1: System Architetcure 

matching:

The following steps take place in ontology

\section{A. Developing Ontologies}

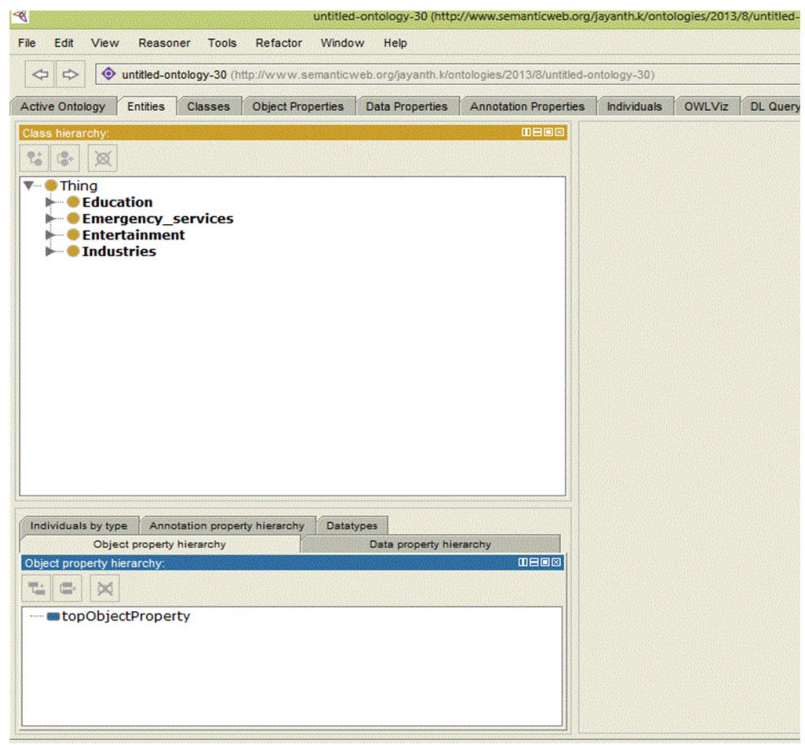

Fig 2: Developing Ontologies

B. Result of Ontology Matching

\begin{tabular}{|c|c|c|c|c|c|}
\hline of & & A Pookge 35 fiecilliest. & 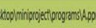 & & \\
\hline 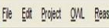 & 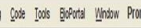 & 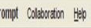 & & & \\
\hline 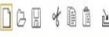 & 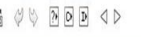 & & & & \\
\hline 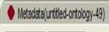 & 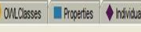 & iass : Fms Proud & & & \\
\hline Toeven Tertiven & & & & & \\
\hline mystatle & & & & & Pits 50 ? \\
\hline 18.30000 & A & terand & qaration & noptera & 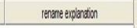 \\
\hline Obciefrils & 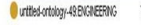 & wos & mpp & Dreath dangend & dittert dohners \\
\hline Obxasion & 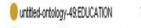 & Yes & Whap & 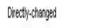 & 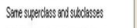 \\
\hline Otrageny & 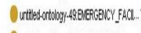 & i.es & map & 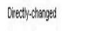 & 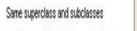 \\
\hline OFfES SITIOI & 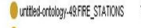 & Yos & $m$ & 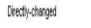 & 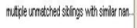 \\
\hline Ohssmals & 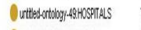 & Yes & map & Drestldanged & Giftert denters \\
\hline Onomel & 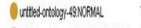 & ise & map & Dresthdranges & dititert dohites \\
\hline OPUU:STAROM & 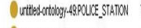 & ises & mp & 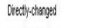 & difterat thenties \\
\hline Oshrows & 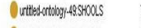 & ves & map & Drestldianged & 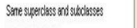 \\
\hline Ostale & 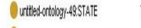 & ves & mp & 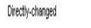 & dititert tomites \\
\hline Qwaienorers & 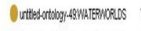 & Yos & w & 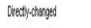 & Githent dethites \\
\hline Inpes inowaty & 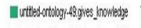 & Yses & map & 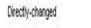 & diftert dethies \\
\hline 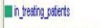 & 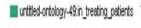 & ives & nop & Dreathdonged & diflestit denters \\
\hline 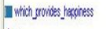 & 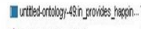 & 1.14es & 能 & 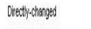 & linersese intily \\
\hline t & 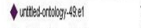 & Yses & Inpap & 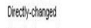 & oiftert dehines \\
\hline 42 & 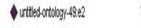 & reses & map & Dreatldianos & 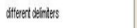 \\
\hline Pow & 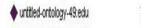 & Yes & Who & 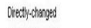 & Giftert dehtites \\
\hline forta & 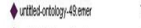 & Yes & map & Drestldatay & Giflyet dohites \\
\hline Petre & 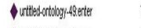 & Yes & mp & Dresth dingest & Giflasert denties \\
\hline Aetri2 & 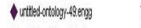 & ves & map & Mrestldanged & lanes se inthity \\
\hline $\operatorname{ton} 2$ & 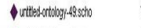 & res & Hap & Drestldianges & lines se seinity \\
\hline tsati & 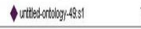 & $y_{0}$ & 栔 & Drentldongen & 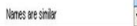 \\
\hline
\end{tabular}

Fig 3: Matched Ontology

\section{CONCLUSION}

In this paper we developed ontologies using protégé and by comparing the developed ontologies we got the similar classes, objects and individuals and also we are able to search the queries by using the DL query it is possible when we start the reasoner The developed ontologies are useful in the future for Merging two ontologies, query processing, Data translation, and for navigation of a web.

\section{REFERENCES}

[1] Hacene Belhadefa, "A new bidirectional method for ontologies matching", proceedings on 2011 International Conference on Power Electronics and Engineering Application

[2] M. Rahamalullah Khondoker, Paul Mueller, "Comparing Ontology Development Tools Based on an Online Survey", Proceedings of the World Congress on Engineering 2010 I WCE 2010, June 30 - July 2, 2010, London, U.K

[3] Gómez-Pérez, A., Fernández-López, M., Corcho, O. (2004) "Ontological Engineering": with examples from the areas of Knowledge Management, e-Commerce and the Semantic Web. 2nd printing, Springer-Verlag, London. ISBN 1-85233-551-3

[4] Jerome Euzenat, Jerome. Euzenat, Jerome Euzenat, Pavel Shvaiko "Ontology matching"Protégé (2000).

[5] Haridimos Kondylakis1, Martin Doerr1, Dimitri's Plexousakis1,"Mapping Language for Information Integration “, Technical Repont 385, ICS-FORTH, December 2006

[6] Dario Salvi, Marco Picone, Mar'1a Teresa Arredondo, Mar'1a Fernanda Cabrera-Umpi'errez, ' Angel Esteban, Sebastian Steger, and Tito Poli, "Merging Person-Specific Bio-Markers for Predicting Oral Cancer Recurrence through an Ontology" ,IEEE transactions on biomedical engineering aT tHE tIME oF 1 , january 2013

[7] Review of ETL and Ontology/Schema Matching Techniques and Tool (2010), By: Geo scientific Data Integration,At:geodi.ucc.ie/files/deliverables/GeoDI_D25_Fina 1.pdf

[8] Thomas R Gruber " A translation Approach to portable Ontology Specifications Knowledge Acquisition",5(2):199220.1993

[9] Fonseca F., Davis C Câmara G."Bridging Ontologies and Conceptual Schemas Geographic Information Integration”, Geoinformatica, Volume 7 Issue 4, 355-378. 2003.

[10] Kalyanpur, Aditya., Parsia, Bijan., Hendler, James "A Tool for Working with Web Ontologies" Proceedings of the International Journal on Semantic Web and Information Systems, Vol.1, No.1, Jan-Mar (2005)

[11] Gruber, T. 1993 "Toward principles for the design of ontologies used for knowledge sharing.", International Workshop on Formal Ontology, 43 (5) pp. 907-928.

[12] S. Steger and G. Sakas, "Image gradient based shape prior for the seg- mentation of not that spherical structures," in Proc.9th IEEE Int. Symp. Biomed. Image, May 2012, pp. 1252-1255.

[13] F.N. Noy and M.A. Musen, "PROMPT Algorithm and Tool for Automated Ontology Merging and Alignment," Proceedings on 17th Nat'l Conf. Artificial Intelligence (AAAI '00), pp. 450-455, 2000. 